\title{
Avaliação da percepção das equipes de saúde bucal da Secretaria Municipal da Saúde de Curitiba (PR) sobre o tratamento restaurador atraumático (ART)

\author{
Evaluation of the perception of the oral health teams \\ of the municipal health department of Curitiba, Paraná State, \\ regarding atraumatic restorative treatment (ART)
}

I vana M aria Saes Busato ${ }^{1}$

M arilisa Carneiro Leão Gabardo ${ }^{2}$

Beatriz H elena Sottile França ${ }^{3}$

Samuel J orge M oysés ${ }^{4}$

Simone Tetu M oysés ${ }^{3}$

${ }^{1}$ Prefeitura M unicipal de Curitiba, Fundação deA ção Social. Rua Eduardo Sprada 4.520, Campo Comprido. 81270-010 Curitina PR. ivanabusato@ibest.com.br

${ }^{2}$ FaculdadeH errero.

${ }^{3}$ Pontifícia Universidade

Católica do Paraná.
Abstract An evaluation was made of the perception of oral health teams regarding Atraumatic Restorative Treatment (ART) as a primary oral healthcare strategy used by the M unicipal $\mathrm{H}$ ealth Department of Curitiba, Paraná State. A study was made both of $\mathrm{H}$ ealth $\mathrm{U}$ nits $(\mathrm{HU})$ that operate using the Family Health Strategy (FHSHU) and those that do not (Basic H ealthcare U nits BHU). The sample involved 191 oral health professionals proportionally $(\mathrm{Cl}=95 \%, e=6 \%)$. A questionnaire was used to collect data. Theinformation was analyzed using the SPSS 13.0 program. The response rate was $82 \%$. Chi-square testing did not reveal a statistically significant difference between the FHSH U $(83.1 \%)$ and the BHU $(74.3 \%)$ with regard to knowledge about the technique $(p>0.05)$. A difference was found as to training in ART among the different $\mathrm{HU}$ $(p<0.01)$ and the use of the technique by the services $(p<0.01)$. The length of training and the length of service of dental health professionals at the municipal health service were relevant in relation to knowledge of the techniqueand the taking of clinical decisions as to ART. The conclusion was reached that training on $A R T$ is needed at the primary healthcare level as a result of the different perceptions revealed.

Key words Primary health care, Family health, Glass ionomer cements
Resumo Avaliou-se a percepção das equipes de saúde bucal sobreo tratamento restaurador atraumático (ART) como estratégia da atenção básica em saúde bucal na Secretaria M unicipal da Saúde de Curitiba (PR). Foram pesquisadas as U nidades de Saúde (US) que trabalham com a Estraté gia da Saúde da Família (USESF) e as que não trabalham (USB). A amostra foi de 191 profissionais proporcionalmente $(\mathrm{IC}=95 \%$, $\mathrm{e}=6 \%)$. Utilizou-se questionário para a coleta de dados. As informações foram analisadas por meio do programa SPSS 13.0. A taxa de resposta foi $82 \%$. 0 teste qui-quadrado não revelou diferença estatisticamente significativa entre as USESF $(83,1 \%)$ e USB $(74,3 \%)$ quanto ao conhecimento da técnica $(p>0,05)$. Diferença foi encontrada quanto à capacitação em ART entre as diferentes U S $(p<0,01)$ e 0 emprego da técnica no serviço $(p<0,01)$. Os tempos de formação e de atuação profissionais na secretaria foram relevantes sobre conheci mento da técnica e tomada de decisão clínica frente ao ART. Concluiu-se que a capacitação sobreART énecessária na atenção básica, em virtude das diferentes percepções reveladas.

Palavras-chave Atenção primária à saúde, Saúde da família, Cimentos de ionômero de vidro 
Introdução

O último levantamento epidemiológico sobre a condição de saúde bucal da população brasileira evidenciou uma situação preocupante ${ }^{1}$. O Brasil atingiu a meta da proposta internacional somente para a idade de doze anos, em que a média de dentes com experiência de cárie (CPO-D) foi de 2,78 (a meta apontava um valor máximo deCPO$\mathrm{D}$ igual 3$)^{2}$. Para outras faixas etárias, os níveis ficaram muito aquém dos objetivos propostos ${ }^{1}$.

Em Curitiba, o quadro epidemiológico vem acompanhando os mesmos padrões brasileiros, apesar dos melhores resultados obtidos em 2003. O CPO-D aos doze anos de idade foi de 1,27. Entretanto, de 35 a 44 anos, 50,16\% apresentaram todos os dentes. Para a faixa etária de 65 a 74 anos, os dados são ainda mais alarmantes: apenas $7,74 \%$ apresentaram vinte ou mais dentes na cavidade bucal ${ }^{3}$.

0 panorama descrito evidencia queo foco da atenção aos problemas de saúde bucal ainda se concentra nos tratamentos restauradores tradicionais, o que leva ao aumento deindivíduos com grandes quantidades de elementos dentários que sofreram algum tipo de interven ção clínica.

N este contexto, a abordagem restauradora tradicional, centrada no tratamento das cavidades, influenciou o crescimento da odontologia restauradora ${ }^{4}$. É consenso a afirmação de que a execução de tratamentos restauradores éfeita isoladamente, sem se considerar a saúde bucal centrada na prevenção $0^{4,5}$.

0 desenvolvimento relativamenterecentedos materiais restauradores minimizou a necessidade deretenções mecânicas. Estes materiais possibilitaram a realização da abordagem de intervenção mínima, que preserva os tecidos dentários, retirando somente as porções desmineralizadas, aumentando, assim, a vida do elemento dentário e evitando o ciclo restaurador repetitivo ${ }^{5}$.

Os materiais restauradores adesivos, como 0 ionômero de vidro, permitiram o desenvolvimento de técnicas restauradoras que preservassem o tecido dentário, como o tratamento restaurador atraumático $(A R T)^{5}$. Este procedimento é indicado por entidades internacionais desde 1994, por ser um método de simples execução e barato, visto que não necessita de aparato tecnológico complex $0^{5,6}$.

A literatura é vasta em estudos que relatam 0 sucesso da técnica-12.

Outro fator de destaque apontado em relação ao material restaurador utilizado no ART éa capacidade de remineralização dentinária ${ }^{13}$.
Os resultados clínicos provenientes da aplicação do ART mostraram resultados bons e apontaram para um futuro promissor, em que uma prática odontológica bem-sucedida deve beneficiar tanto o paciente quanto o profissional ${ }^{5}$.

Assim, dentro do contexto da Saúde Pública, para a implementação plena do Sistema Único de Saúde (SUS) e para o alcance de objetivos e metas, o desenvolvimento de pessoal exige enfoque integrador, interdisciplinar e intersetorial, unindo ensino e serviço ${ }^{14}$. Há responsabilidade do ensino e do serviço em realizar atualizações aos trabalhadores do SUS, com vistas a novas técnicas quesejam baseadas em evidências de efetividade para responder às necessidades de atenção da população.

A Secretaria M unicipal de Saúde de Curitiba (SM S) tem organizado a assistência clínica odontológica pelas estratégias: atendimento programado, pronto atendimento, atendimento à urgência e atenção extraclínica. 0 pronto atendimento (PA) faz parte da atenção básica de saúde bucal edestina-sea atender a queixa principal do usuário e/ou necessidade percebida através delivre demanda ${ }^{15}$.

Neste processo de trabalho, a técnica do ART apresenta-se como uma nova abordagem no controle de cárie dentária, menos traumática, resolutiva ecom vistas a melhorar o pronto atendimento.

Desta forma, o presente trabalho teve por objetivo avaliar a percepção sobre o uso e a ade quação da técnica do ART pelos integrantes das equipes de saúde bucal (ESB) queatuam na atenção básica da SM S de Curitiba.

\section{M étodo}

0 presente estudo caracterizou-se como transversal. A população foi composta por 560 integrantes das ESB, sendo 391 cirurgiões-dentistas (CD) e 169 técnicos em higienedental (THD) que trabalham em Unidades de Saúde (US) da SM S de Curitiba ${ }^{16}$.

Atualmente, a SM S possui 107 US, sendo que, destas, 96 possuem clínicas odontológicas ${ }^{16}$.

Para o cálculo amostral, foi considerado o total de profissionais proporcionalmente entre as duas categorias e US que trabalham com a Estratégia de Saúde da Família (USESF) e que não trabalham com essa estratégia (USB). Trabalhando-se com um IC $=95 \%$ e e $=6 \%$, a amostra compôs-se de 191 profissionais, sendo 133 CD e58THD. 
A avaliação da percepção das ESB sobre o ART foi realizada por meio de questionário autoaplicável com perguntas fechadas, composto de três partes: (1) dados pessoais de formação; (2) informações sobre a técnica avaliada, usando uma escala de avaliação do tipo Likert ${ }^{17}$, de 5 pontos e (3) indicações clínicas do ART.

Os questionários foram distribuídos proporcionalmente entre as US em envelopes com local de remetente de uma central de coleta. Foi crité rio de inclusão a assinatura do termo de consentimento livreeesclarecido.

De posse dos dados, foi montado um banco com todas as variáveis pesquisadas. A análise foi feita com o software deestatísticaSPSS 13.0. N um primeiro momento, foram realizados testes para se verificar a normalidade dos dados (Kolmogorov-Smirnov) ehomogeneidade devariância (Levene). Em seguida, foram realizadas as análises bivariadas pelos testes de qui-quadrado e $U$ de M ann-Whitney, para se verificar diferenças estatisticamente significativas entre os grupos $(p<0,05)$.

Esta pesquisa contou com a aprovação do ComitêdeÉtica em Pesquisa da Secretaria M unicipal da Saúde de Curitiba.

\section{Resultados}

A taxa de resposta obtida foi de $82 \%$. As avaliações da percepção das ESB sobre o ART mostraram que, na primeira parte do questionário de dados pessoais de formação, 52,9\% pertenciam a USESF e $47,1 \%$, à USB. Quanto ao sexo, 76, $4 \%$ foram representantes do sexo feminino e $23,6 \%$, do sexo masculino. Os dados referentes à idade, tempo de formação e tempo de prefeitura não apresentaram distribuição normal, com medianas respectivamente de 39, 15 e 13 anos.
As informaç̧̃̃es sobre a técnica ART mostraram resultados conforme apontado na Tabela 1. A análise de frequências indicou quea ART é conhecida por $79 \%$ dos participantes $(n=124)$, contra $21 \%$ dos que não a conhecem $(n=33)$. Entre as US analisadas, entretanto, não houve diferença significativa nesta questão ( $p>0,05)$.

Quanto à aquisição deste conhecimento durante a formação profissional, somente $21,7 \%$ $(n=34)$ das respostas foram negativas.

Em 36,3\% ( $n=57)$ das respostas, a instituição SM S realizou capacitação sobre ART, contra $31,2 \%(n=49)$ que afirmaram ter recebido capacitação em outra instituição.

A respeito da utilização ou não da ART na atividade profissional realizada na SM S, 65\% $(n=102)$ confirmaram positivamente 0 emprego da mesma.

A Tabela 2 mostra os resultados por questão perguntada em escala tipo Likert. Quando comparadas entre as US, a única questão que apresentou diferença estatisticamente significativa pelo testeU de $M$ ann-Whitney $(p<0,05)$ foi referente ao fato de o ART ser uma técnica restauradora definitiva.

A análise defrequências indicou uma tendência das ESB concordarem quanto ao ART ser uma técnica restauradora que pode ser utilizada na atenção básica de saúde bucal.

Quanto à questão de o ART ser empregado para ampliar o acesso do pronto atendimento, a tendência do grupo ficou entre a neutralidade e concordar com a afirmação. Mesma tendência foi apresentada na afirmação de que a técnica ART deve ser utilizada em dentes permanentes. Contudo, a indicação da técnica para dentes decíduos foi de ampla aprovação nas equipes.

O ART como estratégia de promoção de saúde para controle da cárie dentária apontou na direção de aprovação pelos profissionais partici-

Tabela 1. Avaliação sobre conhecimento, capacitação e emprego da ART pelas ESB da SM S. Curitiba (PR), 2006.

\begin{tabular}{lrrrc}
\hline \multicolumn{1}{c}{ Variável } & $\begin{array}{c}\text { Sim } \\
(\%)\end{array}$ & $\begin{array}{c}\text { Não } \\
(\%)\end{array}$ & $\begin{array}{r}\text { Sem resposta } \\
(\%)\end{array}$ & $\begin{array}{c}\text { Análise bivariada } \\
\text { entre USESF e UBS* }\end{array}$ \\
\hline Conhecimento da técnica ART & 79 & 21 & 0 & $>0,05$ \\
Conhecimento na formação profissional & 21,7 & 68,2 & 10,2 & $>0,05$ \\
Capacitação na instituição SM S & 36,3 & 60,5 & 3,2 & $<0,05$ \\
Capacitação em outro local & 31,2 & 66,9 & 1,9 & $>0,05$ \\
Emprego do ART na SM S & 65 & 33,1 & 1,9 & $<0,05$ \\
Total & 100 & 100 & 100 & \\
\hline
\end{tabular}

*Testequi-quadrado $(p<0,05)$. 
pantes da pesquisa. Ressalta-se que a tendência das equipes foi de concordar que a técnica deve ser amplamente utilizada nas US.

O ART não apresentou aceitação como procedimento restaurador definitivo. Porém, mostrou-se uma tendência afirmativa sobre a importância ao ser usado em clínica odontológica e em ambientes não clínicos.

As indicações clínicas do ART em percentual apresentadas pelos profissionais das ESB estão expostas no Gráfico 1.
Nenhuma das indicações clínicas pesquisadas mostrou diferença entre as U S pelo testequiquadrado $(p>0,05)$.

\section{Discussão}

O ART é indicado como uma técnica efetiva na preven ção e no controle da doença cárie, além de ter baixo custo e reduzir o estresse por parte do paciente ${ }^{18} .0$ mesmo insere-se perfeitamente no

Tabela 2. Q uestões avaliadas so bre o ART em escala tipo Likert dos profissionais das ESB da SM S. Curitiba (PR), 2006.

\begin{tabular}{lcrrrr}
\hline \multicolumn{1}{c}{ Variável } & $\begin{array}{c}\text { Discordo } \\
\text { totalmente }\end{array}$ & Discordo & Neutro & Concordo & $\begin{array}{r}\text { Concordo } \\
\text { totalmente }\end{array}$ \\
\hline ART para atenção básica & $0 \%$ & $1,3 \%$ & $16,6 \%$ & $47,8 \%$ & $34,4 \%$ \\
ART utilizado para ampliar o acesso & $1,3 \%$ & $12,7 \%$ & $23,6 \%$ & $41,4 \%$ & $21 \%$ \\
ART para dentes permanentes & $3,2 \%$ & $15,9 \%$ & $33,7 \%$ & $40,1 \%$ & $7 \%$ \\
ART para dentes decíduos & $0 \%$ & $0 \%$ & $17,2 \%$ & $52,9 \%$ & $29,9 \%$ \\
ART para controle da doença cárie & $0 \%$ & $1,3 \%$ & $14 \%$ & $38,9 \%$ & $45,9 \%$ \\
ART utilizado amplamente nas US & $0 \%$ & $14,6 \%$ & $24,2 \%$ & $38,9 \%$ & $22,3 \%$ \\
ART técnica restauradora definitiva & $14,6 \%$ & $35,7 \%$ & $33,7 \%$ & $13,4 \%$ & $2,5 \%$ \\
ART pode ser utilizado na clínica & $2,5 \%$ & $1,3 \%$ & $17,2 \%$ & $47,8 \%$ & $34,4 \%$ \\
odontológica e em outros ambientes não & & & & & \\
clínicos & & & & & \\
\hline
\end{tabular}

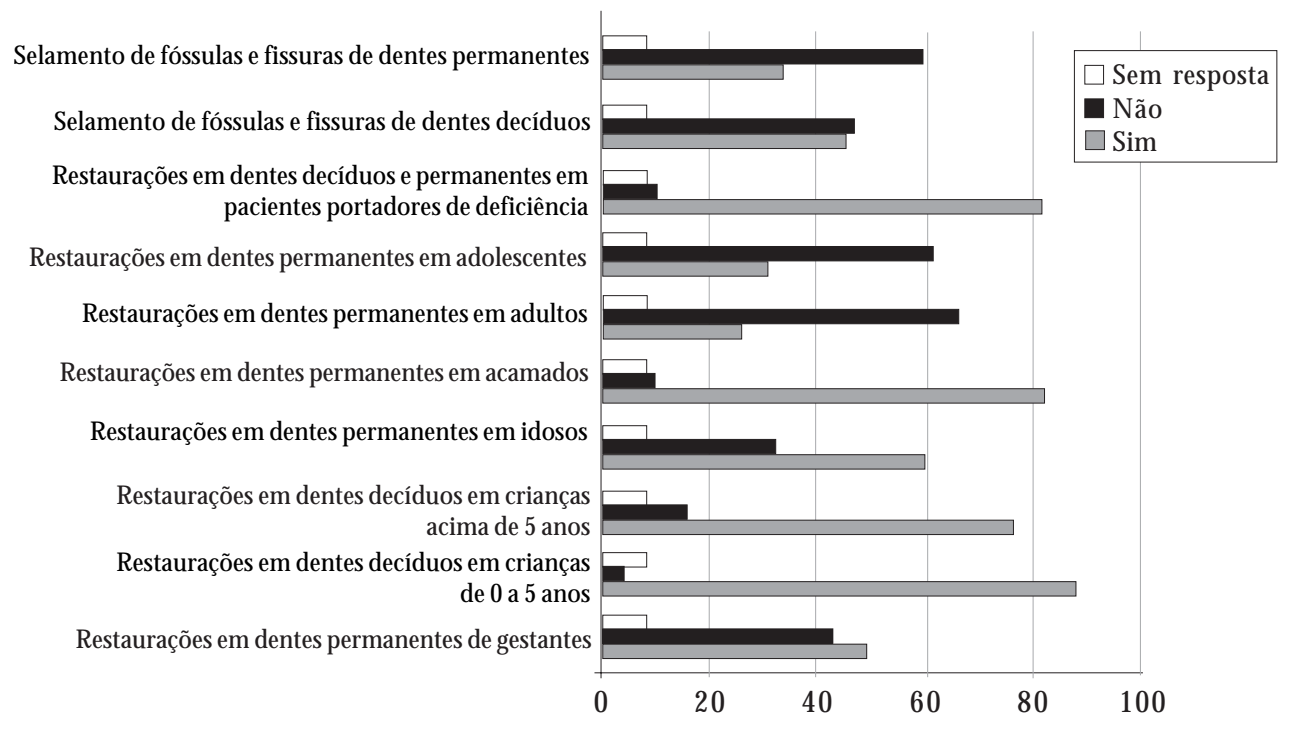

Gráfico 1. As indicações clínicas do ART em porcentagens pelos profissionais das ESB da SM S. Curitiba (PR), 2006. 
atual conceito da abordagem de intervenção mínima, cujo intuito éa preservação detecidos dentários ${ }^{19}$.

Entretanto, em média, os sujeitos desta pesquisa "concordam" ou "concordam totalmente" em apenas $42,2 \%$ que o ART deve ser utilizado no controle da cárie dentária. Isto indica que, apesar de recomendado pela Organização M undial de Saúde ${ }^{6}$, o ART é motivo de discussão no que se refere ao conhecimento da técnica e suas indicações clínicas.

Segundo alguns estudos, o ART não éindicado para restaurações que envolvam mais de uma face, devendo se restringir a cavidades simples, em especial na dentição permanente, e também ao selamento de fissuras, em decorrência das propriedades do cimento deionômero de vidro ${ }^{20}$. Isto vem ao encontro dos resultados da presente pesquisa, sendo que a concordância plena sobre 0 uso do ART na dentição permanente foi indicada por $7 \%$ dos indivíduos.

Em contrapartida, a literatura revela diversos estudos em que as restaurações com cimento de ionômero de vidro tiveram sucesso ${ }^{7-9,11}$.

Comparando-se a qualidade de restaurações declassel entremateriaisconvencionaisem amálgama e restaurações com a técnicaART, foi comprovada que, num período de três anos, a dife rença não ésignificativa para os critérios de qualidade de restaurações ${ }^{10}$.

Em ensaio clínico randomizado, com período deacompanhamento deseisanos, concluiu-seque o ART, comparado com o amál gama, em restaurações de cáries oclusais, tem resultado muito bom e sem diferenças estatísticas para o sucesso das restaurações. Contudo, para o desenvolvimento de cáries secundárias, o ART foi superior. Os autores apontaram um percentual de sucesso de $72,6 \%$ para o amálgama e $72,3 \%$ para o ART ${ }^{12}$.

Em trabalho no Reino Unido, foram distribuídos seiscentos questionários aos cirurgiõesdentistas clínicos gerais, com o objetivo de se de terminar os materiais etécnicas utilizados no tratamento de cáries na dentição primária. A maioria dos entrevistados confirmou utilizar materiais adesivos para restaurarem cáries em molares decíduos; contudo, dos $42 \%$ que afirmaram conhecer o ART, menos de $10 \%$ o realizam ${ }^{21}$. Os dados encontrados na presente pesquisa não se assemelham ao descrito anteriormente, visto que $79 \%$ dos integrantes das ESB da SM S de Curitiba conhecem o ART.

Em estudo recente real izado na Tanzânia, com envolvimento de 117 integrantes das ESB, verificou-se que $35 \%$ dos pesquisados praticam o ART. Entretanto, apenas 6,5\% relataram utilizá-lo "na maioria das vezes" ou "sempre". Os autores concluíram que, frente aos resultados, a perda dentária sobrepõe qualquer outra prática odontológica. Desta forma, a necessidade de expansão do conhecimento e emprego do ART faz-se necessário ${ }^{22}$.

Os achados descritos acima se aproximam dos encontrados neste estudo, pois 30,6\% dos integrantes das ESB "concordam" ou "concordam totalmente" que o ART éamplamente utilizado nas US.

Ainda, quanto à aplicabilidade de diferentes técnicas restauradoras, a aquisição do conhecimento é bastante relevante. Outra pesquisa esclarecequea mudança de critérios e decisões restauradoras está atrelada à necessi dade de treinamentos ou pós-graduação ${ }^{23}$.

Desta forma, o presente estudo propos-se a verificar também a forma pela qual o conhecimento relacionado ao ART foi adquirido. O scursos de capacitação ofertados pela SM S foram indicados por $36,3 \%$ dos indivíduos. Somente $21,7 \%$ afirmaram ter tido conhecimento durante a graduação. Este fato pode ser explicado devido à mediana da fai xa etária ter sido dequinze anos, sendo que o ART surgiu em meados da década de oitenta ${ }^{24}$.

\section{Considerações finais}

O ART vem sendo utilizado pelos profissionais das ESB da Prefeitura Municipal de Curitiba, 0 queevidencia a consonância com os objetivos da atenção básica e da atual Política Nacional de Saúde Bucal para a ampliação do acesso da população ao cuidado em saúde bucal, melhoria dos índices epidemiológicos e aumento da resolutividade da atenção.

Entretanto, os achados deste estudo revelam haver diferentes considerações em relação à técnica, indicações, dentreoutros, o queindica a necessidade de capacitação profissional neste aspecto. 


\section{Colaboradores}

IMS Busato contribuiu para a concepção, coleta dos dados eredação do artigo; M CL Gabardo contribuiu para a análise dos dados e redação do arti- go; BHS França, para a concepção e supervisão do estudo. SJ M oysés contribuiu para a concepção e desenho do artigo, ena revisão crítica do conteúdo eST M oysés, com o conteúdo intelectual do estudo ena aprovação final do texto para publicação.

\section{Referências}

1. Projeto SB Brasil. Condições de saúde bucal da população brasileira 2002-2003. Resultados principais. Braślia: Ministério da Saúde/Secretaria de Atenção à Saúde/Departamento de Atenção Básica/Coordenação Nacional de Saúde Bucal; 2004.

2. Federation Dentaire Internationale. Global goals for oral health in the year 2000. Int Dental J 1982; 32:74-77.

3. Curitiba. Secretaria Municipal da Saúde. Relatório do Projeto SB Brasil Curitiba - 2003. Curitiba: Centro de Epidemiologia; 2003.

4. Elderton RJ. Changing the course of dental education to meet future requirements. J Can Dent Assoc 1997; 63:633-639.

5. Frencken JE, Holmgren CJ. Tratamento restaurador atraumático para a cárie dentária. São Paulo: Santos; 2001.

6. Frencken JE, Makoni F, Sithole WD. Atraumatic restorative treatment and glass-ionomer sealants in a school oral health program in Zimbabwe. Caries Res 1996; 30(6):428-433.

7. Phantumvanit $P$, Songpaisan $Y$, Pilot $T$, Frencken JE. Atraumatic restorative treatment (ART): a threeyear community field trial in Thailand - survival of one-surface restorations in the permanent dentition. J Public Health Dent 1996; 56(3):141-145.

8. Frencken JE, Makoni F, Sithole WD, Hackenitz E. Three-year Survival of one surface ART Restorations and glass-ionomer sealants in School Oral $\mathrm{He}$ alth Programme in Zimbabwe. Caries Res 1998; 32(2):119-126.

9. Lo EC, Luo Y, Fan MW, Wei SH. Clinical investigation of two glass-ionomer restoratives used with the atraumatic restorative treatment approach in China: two-years results. Caries Res 2001; 35(6):458-463.

10. Kalf-Scholte SM, van Amerongen WE, Smith AJ, van $\mathrm{H}$ aastrecht $\mathrm{HJ}$. Atraumatic restorative treatment (ART): a three-year clinical study in Malawi-comparison of conventional amalgam and ART restorations. J Public Health Dent 2003; 63(2):99-103.

11. Frencken JE, van't Hof MA, Van Amerongen WE, Holmgren CJ. Effectiveness of single-surface ART restorations in the permanent dentition: a metaanalysis. J Dent Res 2004; 83(2):120-123.

12. Mandari GJ, Frencken JE, van 't H of M A. Six-year success rates of occlusal amalgam and glass-ionomer restorations placed using three minimal intervention approaches. Caries Res 2003; 37(4):246-253.

13. M assara ML, Alves JB, Brandao PR. Atraumatic restorative treatment: clinical, ultrastructural and chemical analysis. Caries Res 2002; 36(6):430-436.

14. Moysés ST, Sizenando KMC, M enezes SM, Rodrigues I. A Formação e desenvolvimento da equipe de saúde bucal. In: Silveira Filho AD, organizador. Os dizeres da boca em Curitiba. Rio de Janeiro: CEBES, 2002. p. 45-56.

15. Curitiba. Secretaria Municipal da Saúde. Protocolo Integrado de Atenção à Saúde Bucal.Centro de Informação em Saúde. [site da Internet] 2004 [acessado 2006 mar 03]. Disponível em: http://www. curitiba.pr.gov.br/saude/sms/protocolos/bucal.pdf

16. Curitiba. Secretaria Municipal da Saúde. Relatório Comparativo Anual 2004-2005. 2005. Conselho M unicipal de Saúde. [site da Internet] 2004 [acessado 2006 mar 13]. Disponível em: http://www. curitiba.pr.gov.br/saude/sms/relatorio.pdf

17. Mattar FN. Pesquisa de marketing: edição compacta. 3a ed. São Paulo: Atlas; 2001.

18. Tascón J. Restauración atraumática para el control de la caries dental: historia, características y aportes de la técnica. Rev. Panam. Salud Públ. / Pan Am. J. Public Health 2005; 17(2):110-115.

19. Lima DC, Saliba NA, M oimaz SAS. Tratamento restaurador atraumático e sua utilização em saúde pública. RGO 2008; 56(1):75-79.

20. Smales RJ, Ngo HC, Yip KH, Yu C. Clinical effects of glass ionomer restorations on residual carious dentin in primary molars. Am J Dent 2005; 18(3):188-193.

21. Burke FJ, M CHugh S, Shaw L, Hosey MT, Macpherson L, Delargy S, Dopheide B. UK dentists' attitudes and behaviour towards Atraumatic Restorative Treatment for primary teeth. Br Dent J 2005; 199(6):365369.

22. Mandari GJ, M atee MI. Atraumatic Restorative Treatment (ART): the Tanzanian experience. Int Dent J 2006; 56(2):71-76

23. Maggs-Rapport FL, Treasure ET, Chadwick BL. Community dental officers' use and knowledge of restorative techniques for primary molars: an audit of two Trusts in Wales. Int J Paediatr Dent 2000; 10(2):133-139.

24. Frencken JE, Van Amerogen $E$, Phantumvanit $P$, Songpaisan $Y$, Pilot T. M anual for the atraumatic restorative treatment approach to control dental caries. $3^{\text {rd }}$ ed. WHO Groningen: Collaborating Centre for Oral Heath Services Research; 1997.

Artigo apresentado em 16/06/2008

Aprovado em 15/01/2009

Versão final apresentada em 20/02/2009 